\title{
LSB based Steganography Techniques for Secured Communication
}

\author{
Sahil Kaushal \\ M.E., Wireless Communication \\ Thapar Institute of Engineering \& Technology
}

\author{
Ajay Kakkar \\ Assistant Professor, ECE Dept. \\ Thapar Institute of Engineering \& Technology
}

\begin{abstract}
Steganography is a data hiding technique which is used to prevent the data or any relevant information from falling to the hands of an unauthorized personal. So, in order to prevent such scenario the steganographic method is used using LSB matching technique in which the LSB of the pixels of the cover media is replaced with that of the relevant information and the cover media is chosen based on characteristics known to only the sender and receiver and no one else can access it. The steganographic technique can find its relevance and scope in merely many field like Marketing, MNC's, Industry. The literature review has been done by keeping in mind to obtain ideas and techniques to detect and improve as much as possible to the steganographic techniques and through research and work done on MATLAB software, were able to detect LSB based steganographic encrypted image from the others. To this some remarks were added to improve the security and evident in result section.
\end{abstract}

\section{Keywords}

Steganography, Communication

\section{INTRODUCTION}

The most basic definition of a security system can be defined as a system which is responsible for providing and maintaining the security measures for any system to whom it is assigned to or responsible for example home security system, car security system etc [3].

\section{Cryptography}

It can be referred as an art or technique of hiding any form of important data or information from being assessed by any unwanted or unauthorized user. The encrypted text is known as cipher text, and in order to retrieve the text back the receiver needs the key which is only meant to be shared with the authorized users [7]. During this process data or information is encrypted into cipher text format with the help of the a secret key, and the secret key is only shared with only the authorized person so that only they are able to decrypt the data or information using the same key which is used to encrypt the data or information [9]. Through the presence of the steganography could be traced in the history literatures. Three of these techniques were precisely interconnected like cryptography, steganography and watermarking as shown in figure 1.7 .

\section{LITERATURE SURVEY}

Mehdi Boroumand et.al. [1] demonstrated that express nonstraight element maps combined with straightforward classifiers and enhanced the precision of current steganalysis indicators worked as two fold classifiers and also quantitative identifiers as payload regressors. The non-linear map acquired had little dependency on cover and low computational complexity this technique help to reduce error. Bin Li et.al. [2] proposed an adjusted LBP form, called limit LBP (TLBP), to uncover the antiques caused by information installing. In this steganalytic conspire, the TLBP task was performed on leftover pictures which were gotten by utilizing an arrangement of high request subordinate filters to catch complex connections among pixels. Yun-Te Lin et.al. [3] introduced an algorithm which exploited each three 10-bit mantissa as an embedded unit for concealment of $k$ bits of a hidden message using a favorable base that provides the least pixel variations. This purpose, an expert encoder and decomposition scheme was suggested, that offered a high probability of transmission of $k+1$ bits without causing an increase in pixel variation caused by message concealment. Jishen Zeng [4] developed a generalized hybrid learning model of JPEG format steganalysis integrate the domain knowledge for the construction of an affluent steganalytic models. There were two main stages involved in the proposed framework. The initial stage was related to the convolution of phase and the quantization and truncation of phase of the affluent model. The successive stage was a gathered deep neural network consisting of multi deep subnets for which the modeled parameters were learned during the training procedures. Alsharif Abuadbba et.al. [5] suggested that for maximum hiding, fast Walsh-Hadamard transformation was utilized for this transformation of signals were grouped and the signal having lowest distortion coefficients, was employed. The purpose of upgrading of security, the key was deployed in three-dimensional (3-D) faction of random coefficient for the concealment of the process. The resultant distortion was measured within all the stages. Jan Camenisch et.al. [6] proposed an ideal functionality for non-committing encryption with locally generated, and therefore noninteractive, cipher texts. As a sanity check, they also provided a property based security notion that that proved to be equivalent to the universal composability framework that enables the modular design of cryptographic protocols by allowing arbitrary compositions of lower-level building blocks. Tomas Denemark et.al. [7] defined a substitute kind of side information after examining a course of action of different JPEG photos of a comparable scene for applications when the sender does not approach a pre-cover. The additional JPEG pictures were used to choose the favored furthest point of embeddings changes to adjust the costs of changing individual DCT coefficients in a current embedding plan. Kaibin Huang et.al. [8] introduced a dual-server PEKS (DS-PEKS) syntax to deal with this issue. There were front server and back server in their architecture and the keyword search test was done by the cooperation of two servers. Assumed that these two servers do not collude, the DS-PEKS scheme would be secure against offline inner keyword guessing attacks. Qinglei Kong et.al. [9] proposed a secure handover session key management scheme via mobile relay in networks. Specifically, to achieve forward and backward key separations, the session key shared between the on-board user equipment and the connected donor evolved nodes. It is first generated by the on-board user equipment and then securely 
distributed to the sub-nodes. Mimi Ma et.al. [10] worked for development of a new well secured channel with a certificateless searchable public key encryption standard with different keywords for host establishment.

\section{OBSERVATIONS}

As previous approaches described were not up to date in terms of standards for the present day security requirements but it was quite helpful in defining some of the flaws where it can be improved. It have been provided with suitable advancements which saw were need for raising its standards described with following advances:

- Providing of encryption of date previously before initiation of its embedding process. Decision and selection of suitable covers which provide high attributes of colors for raising the noise of the image so as to disguise or hide the little bit significant changes done in the cover doesn't draw any attention.

\section{COMPARISON}

Image steganography is the craftsmanship and study of hiding a message in a picture by altering picture pixels as well as recurrence coefficients. The most vital necessity in steganography is imperceptibility. Accordingly, different steganographic strategies endeavor to insert messages in an indistinct way with the goal that the subsequent stego is like its relating spread picture outwardly and factually. LSB substitution is the most straight forward steganographic technique. Nonetheless, it brings some asymmetry antiques into stegos, and along these lines it is effortlessly recognized utilizing some steganalytic techniques, for example, Chisquared assault [11], normal/solitary gathering investigation [10] and test combine examination. LSB coordinating was then prepared to evacuate asymmetry ancient rarities presented by LSB substitution by means of arbitrarily adding \pm 1 to pixel values. Contrasted with LSB substitution, LSB coordinating enhances undetect capacity significantly. In this manner, some run of the mill strategies, for example, LSB coordinating returned to and PVD [3], were additionally included later. The above techniques can be viewed as nonversatile steganography, which implies that the modified pixels after information covering up would be arbitrarily spread over the entire picture. Nonetheless, it is demonstrated that pixels situated in textural districts have much preferred concealing properties over those in smooth locales, and this reality is utilized as a part of some versatile steganography. Both cover image and sender's database appropriations amid the implanting procedure, which enhances the security.

\section{Versatile Steganography restriction}

analysis: In this section, common embedding properties are demonstrated of versatile steganography, and afterward the breaking down the confinement of versatile steganography in view of the installing probabilities.

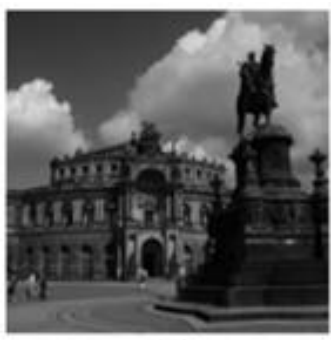

(a)

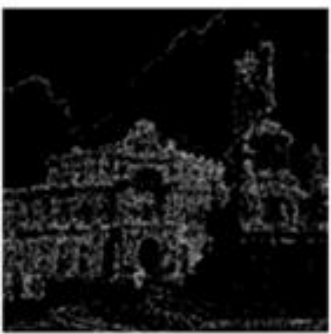

(c)

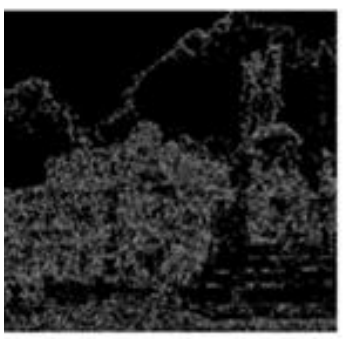

(e)
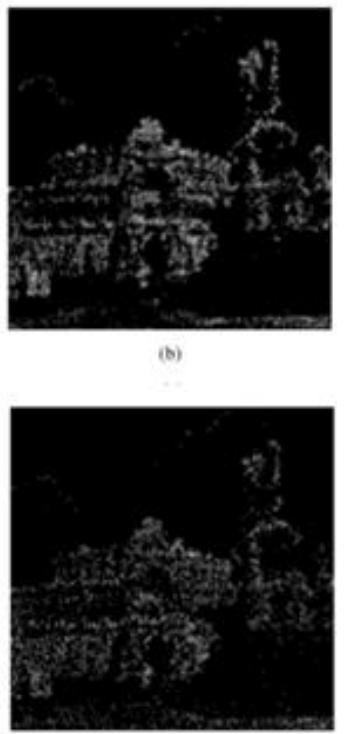

(d)

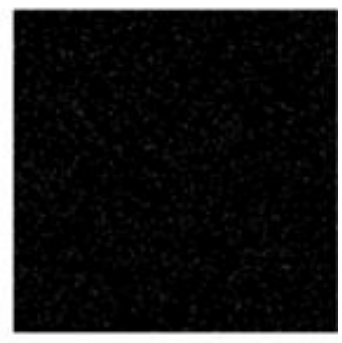

Figure 4.1 Delineation of cover picture and the comparing modifications utilizing WOW, HUGO BD, S-UNIWARD, EA, and LSB coordinating (0.3bpp). (a) Cover Image. (b) WOW. (c) HUGO BD. (d) S-UNIWARD. (e) EA. (f) LSB matching

\section{Embedding property of Steganography}

Fig 4.1 demonstrates a case of a cover picture and the relating modification outline five average steganographic strategies, including WOW, HUGO BD, S-UNIWARD, EA, and LSB coordinating, with the same inserting rate of $0.30 \mathrm{bpp}$. It can be seen that the areas of the modifications change for various versatile techniques. The purpose behind the distinctions is that different versatile steganography would apply various installing methodologies. For LSB coordinating, the modified pixels were haphazardly situated in the entire picture. For the EA strategy, sharp edge areas were firstly considered for information covering up. While the other three techniques (i.e., WOW, HUGO BD, and S-UNIWARD) were composed under the structure of limiting a defined twisting capacity, as outlined in figure 4.3. In this system, each pixel was first allocated an installing cost, which speaks to how much contortion it takes to change a specific pixel, at that point a twisting capacity was defined in view of the inserting costs, and finally some coding procedures, for example, STCs, were utilized to limit the mutilation work and acquire the subsequent picture stegos. It should be noted that one of the fundamental contrasts between steganographic strategies under such a structure was the plan of the inserting cost. 


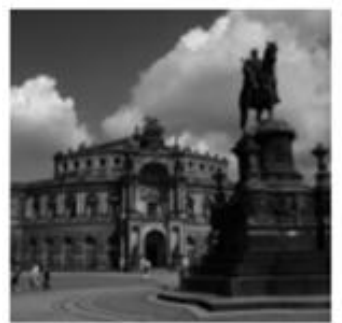

(a)

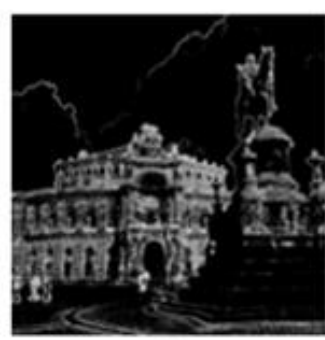

(c)

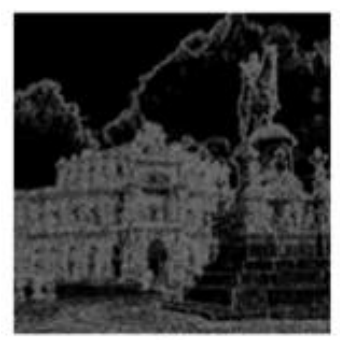

(e)

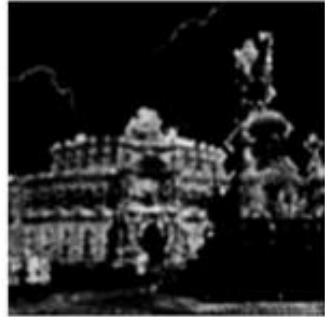

(b)

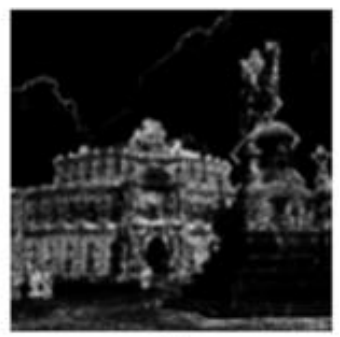

(4)

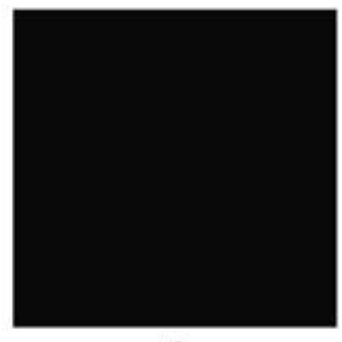

in
Figure 4.2 Outline of cover picture and the comparing inserting probabilities/likelihoods with WOW, HUGO BD, S-UNIWARD, EA, and LSB coordinating (0.30 bpp). (a) Cover Image. (b) WOW. (c) HUGO BD. (d) S-UNIWARD. (e) EA. (f) LSB matching.

For example, in ASO [22], the implanting cost was the total of all perceptibility costs acquired from various FLD classifiers. In HUGO [17], the installing cost was figured as the separation between the SPAM highlight separately removed from the cover and the stego. HUGO BD [24] was an enhanced variant of HUGO and its inserting cost thinks about the collaborations of installing inside a nearby neighborhood. In WOW [8], the installing cost was computed as the conglomeration of the progressions of various directional high-pass wavelet filters. For S-UNIWARD [9], the implanting cost was ascertained as the total of the relative changes of the coefficients in the wavelet filter banks and so on. Fig 4.2 (b)- (e) indicate likelihood maps relating to Fig 4.1 (b)- (e). It should be further noted that the scale installs probabilities in a scope of $[0,1]$ to a scope of $[0,255]$ for show purposes, and Figure 4.2 (e) was somewhat dim because of the installing likelihood of every pixel being near zero. It would be ideal if looked at the modifications in Figure 4.1 and the relating inserting probabilities in Figure 4.2 beneath. It was discovered that in spite of the fact that the modifications were diverse for various versatile steganography, the regular characteristic they shared were that the modification outline fundamentally were the same as its corresponding likelihood. Examining the connection between the modification guide and likelihood guide, and attempt to uncover the normal steganography.

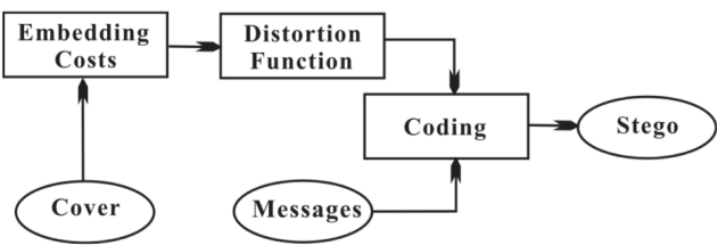

Fig 4.3 Versatile steganography technique in view of the structure of limiting the distortion function

5. RESULTS

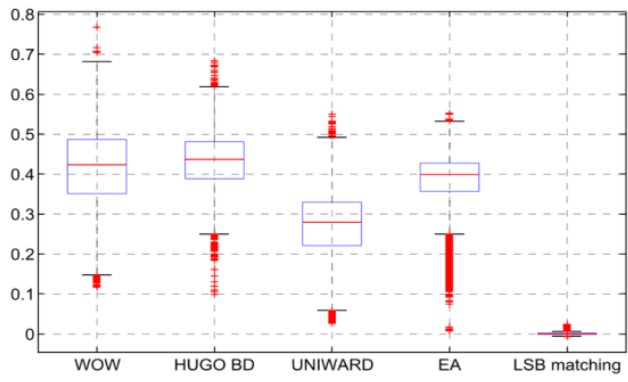

Fig 5.1 Box plot of the correlation coefficients for different steganography

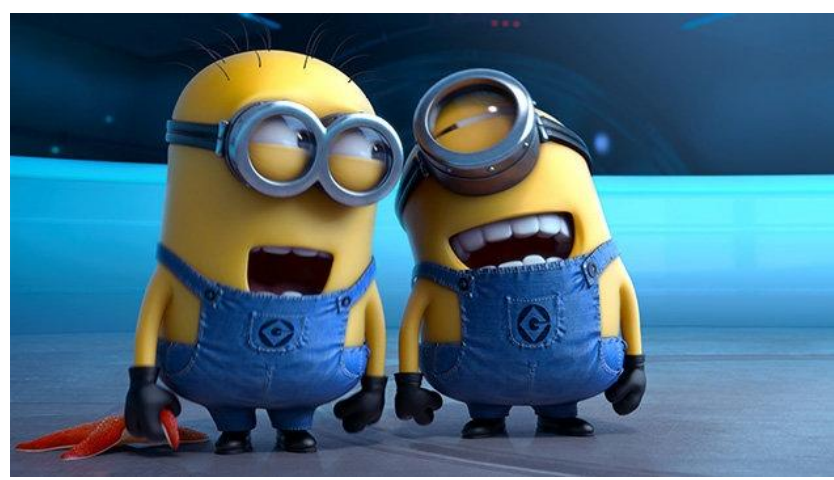

Figure 5.2:Original image

The figure 5.2 is the original image without any kind of embedding of any secret message. It is very important part for the steganographic process as poor selection of any cover image can lead to suspicion and detection of the secret message turning the whole process to trash (preferable a moderate size image with high color ratio).

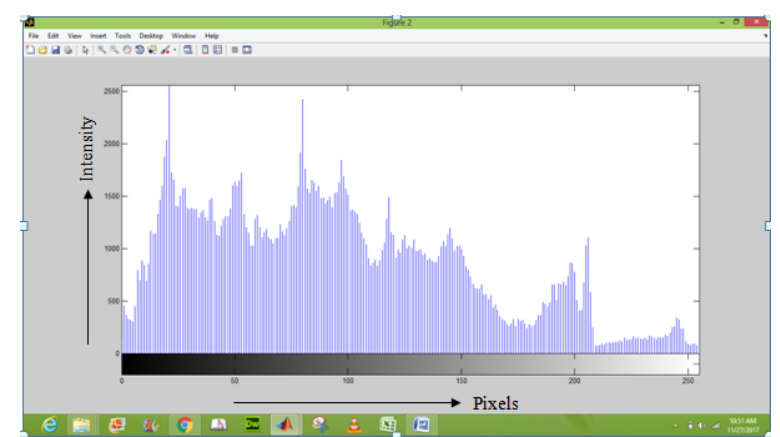

Figure 5.3:Histogram of original image

The figure 5.3 depicts the histogram of the original image with its corresponding characteristic curve of pixel to intensity values. This is the original curve of the image it can be seen it has high difference values between peak to pit values. 


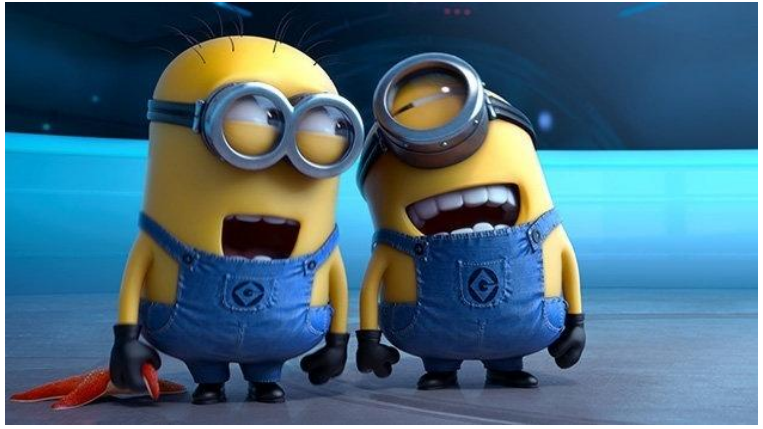

Figure 5.4: Secret message embedded image (stego-image)

The figure 5.4 depicts the stego-image or the embedded secret message image, this is the final processed version of the original image as told earlier the image choose should be of moderate size and high color ratio so that the minor or little change done in the image during embedding could be cover with already existing colored noise of the image. Causing least or none of suspicion to it.

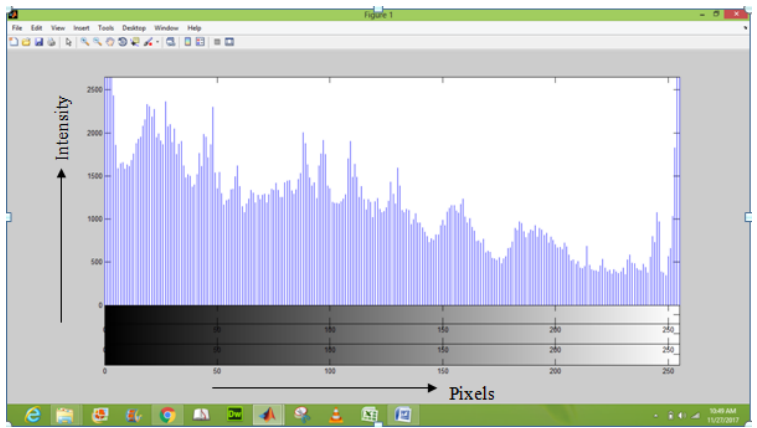

Figure 5.5: Histogram of stego-image

The above figure 5.5 depicts the histogram of the embedded stego-image. Here the histogram is not a single variant histogram. The histogram presented in the figure 5.3 is a convolution of all the three colors of the basic spectrum of light (red, green and blue variants respectively). Need of this convoluted form is because the data embedding is done uniformly in all the three planes. If any one variant will be missing, it won't make any sense on the retrieval of the information because some part which rests in the missing variant won't be there. As seen above the peak to pit value difference is lesser in this histogram as compared to the previous one in figure 5.5. This is because the embedding is done within these peak to pit values to minimize any type of detectable change.

\section{CONCLUDING REMARKS}

Based on the information and results from simulation result from section 5 it can be concluded that LSB based steganography technique is one of the most simple and economic technique as compare to others and it is far more suitable for communicating in commercial targets with not so high priority. In case of high value target achievement it is needed to make so remarks to improve its performance and scale of security. The following are some remarks which can be added:

- Encrypting of message pre from coding and transmitting so that anyone who decodes it must also decrypt it before it makes any sense. Pseudo random noise is also be added to it which makes it impossible to decrypt without have the type of pseudo-random noise generator and it corresponding peers and algorithm. File size is to be kept a minimal magnitude, so as the embedding could be swift, effective and undetectable for general purposes 8 bit image should be employed rather than large magnitude media as it would cause suspicion.

\section{REFERENCES}

[1] M. Boroumand and J. Fridrich, (2018) "Applications of Explicit Non-Linear Feature Maps in Steganalysis," IEEE Transactions on Information Forensics and Security, vol. 13, no. 4, pp. 823-833.

[2] B. Li, Z. Li, S. Zhou, S. Tan and X. Zhang, (2018) "New Steganalytic Features for Spatial Image Steganography Based on Derivative Filters and Threshold LBP Operator," IEEE Transactions on Information Forensics and Security, vol. 13, no. 5, pp. 1242-1257.

[3] Y. T. Lin, C. M. Wang, W. S. Chen, F. P. Lin and W. Lin, (2018)"A Novel Data Hiding Algorithm for High Dynamic Range Images," IEEE Transactions on Multimedia, vol. 19, no. 1, pp. 196-211.

[4] J. Zeng, S. Tan, B. Li and J. Huang, (2018)"Large-Scale JPEG Image Steganalysis Using Hybrid Deep-Learning Framework," IEEE Transactions on Information Forensics and Security, vol. 13, no. 5, pp. 1200-1214.

[5] Abuadbba and I. Khalil, (2017) "Walsh-HadamardBased 3-D Steganography for Protecting Sensitive Information in Point-of-Care," IEEE Transactions on Biomedical Engineering, vol. 64, no. 9, pp. 2186-2195.

[6] J. Camenisch, A. Lehmann, G. Neven and K. Samelin, (2017) "UC-Secure Non-interactive Public-Key Encryption," IEEE 30th Computer Security Foundations Symposium (CSF), Santa Barbara, CA, pp. 217-233.

[7] T. Denemark and J. Fridrich, (2017) "Steganography With Multiple JPEG Images of the Same Scene," IEEE Transactions on Information Forensics and Security, vol. 12, no. 10, pp. 2308-2319.

[8] K. Huang and R. Tso, (2017) "Provable secure dualserver public key encryption with keyword search,"IEEE 2nd International Verification and Security Workshop (IVSW), Thessaloniki, pp. 39-44.

[9] Q. Kong, R. Lu, S. Chen and H. Zhu, (2017) "Achieve Secure Handover Session Key Management via Mobile Relay in LTE-Advanced Networks," IEEE Internet of Things Journal, vol. 4, no. 1, pp. 29-39.

[10] M. Ma, D. He, N. Kumar, K. K. R. Choo and J. Chen, (2017) "Certificateless Searchable Public Key Encryption Scheme for Industrial Internet of Things," IEEE Transactions on Industrial Informatics, vol. no. 99 , pp. 1-1. 